\title{
Literatrónica: un análisis hipertextual de Condiciones extremas, de Juan B. Gutiérrez'
}

\author{
E-Literature: a Hypertextual Analysis of Condiciones extremas, \\ by Juan B. Gutiérrez \\ JOSÉ MARÍA GARCÍA LINARES \\ IES Güímar \\ España \\ kaluitas@yahoo.es
}

(Recibido 26-II-2OI2; aceptado o7-O9-2OI3)

Resumen. Este artículo aborda el análisis de la obra hipertextual Condiciones Extremas, de Juan B. Gutiérrez, desde una perspectiva hipertextual. Para ello se tendrá en cuenta no solo el contexto socio-cultural en el que surge, sino también cuestiones teóricas fundamentales para este tipo de obra como la nomenclatura, la nueva concepción de autor y lector y la del texto como juego.

Palabras clave: Hipertexto; Condiciones Extremas; Autor; Lector; Juego.
Abstract. This article addresses the analysis of the hypertextual work Condiciones Extremas, by Juan B. Gutierrez, from a hypertextual perspective. For that purpose we will take into account not only the sociocultural context in which it arises, but also the theoretical issues basic for this type of work, such as nomenclature, the new conception of writer and reader or the one that accounts for the text as a game.

Keywords: Hypertext; Extreme Conditions; Writer; Reader; Game.

\footnotetext{
I Para citar este artículo: García Linares, José Ma (20I4). Literatrónica: un análisis hipertextual de Condiciones extremas, de Juan B. Gutiérrez. Alabe 9. [www.revistaalabe.com]
} 


\section{I - Introducción}

La llamada revolución tecnológica puesta en marcha en las décadas finales del pasado siglo ha obligado a repensar los cimientos de la cultura: el concepto mismo de libro, la percepción y concepción del saber, el ámbito de la creación... Internet ha generado un cambio sociocultural que introduce interactividad, multiplicidad de itinerarios, universalidad y alteridad ${ }^{2}$.

Dicha revolución ha inclinado la cultura más hacia el lado de su gran capacidad de almacenamiento, distribución y uso que no del de la propia creatividad. Utilizar un ordenador como mediador de lectura, a través de la pantalla, es absolutamente diferente que emplearlo como instrumento de creación. Analizando la historia del hipertexto sin apasionamientos y con los pies en el suelo, tras una etapa inicial de esplendor parece estar sumido en un cierto estado de estancamiento ${ }^{3}$ que los creadores no saben muy bien cómo resolver, afirmando su supervivencia en el modo de leer y de escribir aunque sin ser esa estructura dominante de la web que anunciaron los primeros defensores, sino una más de entre las que existen.

Como sostiene Virgilio Tortosa (2008), sólo en el momento en el que el artista se plantee los límites de la nueva textualidad será capaz de generar una obra a la altura del nuevo medio.

Aunque la tecnología se hace ya tan visible que deslumbra, es decir, sus formas están por encima de cualquier tipo de contenido ${ }^{4}$, nuestros límites como usuarios se reducen en la práctica a leer en pantalla libros tradicionales, reproducidos digitalmente, donde el uso propiamente hipertextual se ve bien reducido por las propias limitaciones del medio quizás por los modos de modulación de la mente humana, lo que significa luchar contra una tradición milenaria 5 .

Por otra parte, dejando de lado a los pesimistas y apocalípticos de las nuevas tecnologías y a los que han querido, a su vez, ver en éstas la salvación del arte con sus teorías mesiánicas, parece que la clave del debate puede residir en las ideas de convivencia y de hibridación ${ }^{6}$. En el terreno artístico, los más diversos discursos, desde la publicidad al cine, desde la performance teatral a los videojuegos, desde la narrativa a la poesía, etc., todo apunta a un espacio abonado para la intertextualidad y la mezcla de géneros?

\footnotetext{
${ }^{2}$ Véanse Aguirre Romero, J. Ma . (2OII), Borràs Castanyer, L. (2OII), Sánchez Mesa Martínez, D. (2OII) y Tortosa, V. (2008).

3 Frente a la postura mesiánica que defendiera Landow, R. (2008) a propósito de las virtudes y milagros del hipertexto, hoy predomina un posicionamiento más realista en cuanto a las posibilidades de la hipertextualidad aplicada al campo de la literatura, como sostiene Pajares Tosca, S. (2004).

${ }^{4}$ Argumento, ya clásico, defendido por Mcluhan, M. (2009).

5 No obstante, empiezan a popularizarse nuevos productos literarios para cuya lectura es necesaria una interface. Nos referimos, por ejemplo, a aplicaciones para iPad del tipo iPoe con la que podemos acceder a una edición digital de ciertos cuentos de Poe en los que se mezclan el audio, animaciones y el texto.

${ }^{6}$ Véase la introducción de Tortosa, V. (2008).

7 Junto a esa mezcla de géneros hoy asistimos igualmente a la mezcla de medios, a la cultura de la convergencia que defiende Jenkins, H. (2008) o a las llamadas narrativas transmedia, como sostiene Scolari, C. A. (2OI3).
} 
Las nuevas tecnologías están definiendo un nuevo espacio social de interrelaciones humanas. Dicho espacio, denominado por Javier Echeverría (2003: 15) "tercer entorno", se opone a los otros dos espacios sociales anteriores que son la naturaleza y la ciudad.

Este nuevo entorno sólo es posible en sociedades de alto desarrollo científico y tecnológico o en determinadas regiones en vías de desarrollo y se define por su interacción y a distancia. Establece una nueva estructura, la de la sociedad de la información, y un funcionamiento de la esfera social completamente distinto.

Frente a la cultura del, vamos a llamarlo así, primer entorno, basada ante todo en el habla y la presencia del cuerpo, o del segundo, que requiere una plasmación material de los objetos culturales, tales como la escritura, los libros, la pintura, la cultura digital del tercer entorno está basada en representaciones tecnológicas digitalizadas a las que se puede acceder a través de redes telemáticas.

También las identidades se han ido desarrollando en cada uno de estos espacios sociales de manera distinta. En el entorno de la naturaleza la identidad es sobre todo física y biológica, enclavada en unas coordenadas espacio-temporales precisas. En el segundo entorno, propio del desarrollo de las ciudades y de sociedades industriales, a esta identidad se le superpone otra más social o civil. El hombre no se define sólo por el quién es (nombre, apellidos, lugar de origen), sino también por el qué hace socialmente. En el tercero, la nueva identidad es, ante todo, digital y numérica, y requiere de medios tecnológicos, científicos y económicos para configurarse. Es una tele-identidad que no requiere presencia física sino de herramientas informáticas, y al ser representación del sujeto que hay detrás, es susceptible de cambiar con facilidad.

La falta de ubicaciones precisas es lo que da pie a Jenaro Talens (2007: 46) para hablar de Internet como "la metáfora de una ciudad sin mapa", llena de caminos, de conexiones, pero sin señales indicadoras que nos ayuden a orientarnos por sus calles.

Este ciberespacio y el hipertexto son hijos de la era del simulacro, de la cultura emergida en la era digital, caracterizada por la falta de armonía, la ruptura de cualquier orden representacional, una cultura que no supone la ruptura drástica con la anterior, sino que crece en un mundo virtual sin necesidad de desmontar lo ya generado. Estudiosos como McLuhan o Bolter han demostrado que los libros son el primer y más lejano artefacto tecnológico, una máquina para ser usada y un producto para ser compartido y vendido.

Como dice Virgilio Tortosa (2008: 9I-97), la cultura hipertextual ha sido comparada con la lógica interna del cerebro humano por ser azarosa, conectiva, caótica y asociativa. A lo largo de la historia el ser humano ha ido poniendo en orden ese caos porque parecía ser el mejor modo de organizar una sociedad. Imponer un orden a la realidad parecía el modo más natural de imprimir una personalidad humana a las cosas. Sin embargo, quedó demostrado con los avances de la neurociencia que la mente del hombre genera pensamientos a través de ligazones o conexiones disímiles. 
La discontinuidad, la simulación dinámica, la interactividad, la fluidez, la descentralización, la libertad creadora, la multilinealidad, el juego, la diversidad...son todas propiedades del hipertexto ${ }^{8}$ que han generado unas transformaciones en los roles y en los agentes que intervienen en la cultura como nunca antes había ocurrido, porque lo definitorio de esta nueva escritura es, precisamente, su naturaleza digital, el realizarse en un espacio digital. No olvidemos que a lo largo de la Historia se produjeron textos que ya tenían alguna de estas características, como demostró Belén Gache (2006).

Los estudios de la literatura electrónica se han multiplicado en la última década. Después del recorrido que hemos realizado a lo largo del trabajo, nos gustaría proponer una clasificación de la mano de Francisco Chico Rico (2008: IO4-IO6), que a su vez sigue las propuestas de Jan Baetens (2003), para las aportaciones teóricas en este campo. El primero de los grupos, en donde encontraríamos trabajos sobre las nuevas tecnologías y los nuevos medios electrónicos de comunicación social en general, estaría representado por autores como Marshall McLuhan, Jay D. Bolter entre otros. En su marco, la ficción literaria no parece ser condenada al olvido, sino sometida a una redefinición sobre la base del poder y de las oportunidades que a aquélla le confieren la implantación y el desarrollo de las nuevas tecnologías de la escritura. La Literatura en sí misma desempeña sólo un papel menor en este tipo de investigaciones, donde el hardware continúa siendo visto como el eje principal de estudio y análisis.

El segundo grupo, de estudios sobre los nuevos medios electrónicos de comunicación social desde un punto de vista más filosófico o teórico-crítico, estaría representado por autores George P. Landow, Michael Joyce o John Tolva. Ahora, la literatura electrónica es aceptada como el equivalente literario en la era tecnológica de la literatura oral y escrita tradicional.

Por último, un tercer grupo de estudios culturales contemporáneos aplicados a la literatura electrónica y/o a la cultura electrónica, representado por autores como Katherine Hayles. En su marco, la literatura electrónica, más que un dispositivo técnico, más que una forma y un contenido específicos, más que un nuevo contexto institucional, más que la experiencia extrema de proximidad, de inmersión, de interactividad, sería el conjunto total de todos esos elementos cambiantes.

Uno de los puntos álgidos del debate se ha centrado en las figuras del autor y del lector. Condicionadas en un primer momento por las teorías postestructuralistas, hoy puede afirmarse que la ficción interactiva no plantea la desaparición del autor, ni del narrador, sino más bien la apertura de alguna de sus funciones a un lector con avidez de participar en el proceso narrativo de un modo más activo, sin que ello lo eleve a la categoría de autor. Para Bobes Naves (2008: I74), el autor no es un individuo cualquiera. Respecto

\footnotetext{
${ }^{8}$ Para el concepto de hipertexto, sus características y precedentes, véanse Aarseth, E. (2004), Borrás Castanyer, L. (Ed.) (2OO5), Cassany, D. (2OI2), Goicoechea, M. (2OO7), Landow, G. (2008), Mendoza, A. (2OIOa, 2OIOb, 2OI2a, 2OI2b) Moreno Hernández, C. (I998), Moulthrop, S. (2003), Orozco Vera, M. J. (2005), Pajares Tosca, S. (2004), Romera Castillo, J. (i996), Romero López, D. y Sanz Cabrerizo, A. (Eds.) (2008). Ruiz Domínguez, Ma M. (2OI2), Tabernero, R. (2OI2), Tortosa, V. (2008).
} 
a la obra literaria es el creador de un mundo en el que se expresa y deja sus huellas. El lector, en cambio, puede ser cualquiera, sin más mérito que saber leer. El esquema básico autor-mensaje-lector es necesario para reproducir el proceso real de comunicación literaria. La diferencia entre un científico de la naturaleza y un científico de la literatura es que la obra literaria tiene un autor, cuya presencia en el objeto condiciona la investigación.

A pesar de la novedad del medio, el hipertexto no reconfigura el paradigma literario ni revoluciona, a día de hoy, la enseñanza de la literatura como predijeron los primeros teóricos. Su importancia se ha ido diluyendo de manera que la teoría literaria digital está buscando nuevos aspectos que investigar, relacionados con la naturaleza multimedia de de la literatura digital o la conectividad. El interés de autores y lectores por las obras de ficción hipertextuales se ha trasladado a la experimentación con otras áreas, como la imagen o el sonido, y en el terreno de la enseñanza, el hipertexto sirve como complemento de la educación tradicional, no como sustitutivo.

Parece, pues, necesario plantear el debate en otros términos, procurando analizar el hipertexto no en lo que tiene de semejante a los presupuestos teóricos de los años setenta y ochenta, sino en lo que es y significa en la sociedad de la información y la comunicación. Como defiende María Goicoechea (2007: I7-2I), necesitamos proponer un nuevo paradigma que dé respuestas fiables, contemporáneas, a esa cultura de lo hiper para ver qué tiene que ofrecer al estudio de la literatura. Un paradigma no constituye sólo un referente, un conjunto de criterios que los lectores usan para juzgar la literariedad de los textos, sino también una batería de preguntas, cuestiones, que hacen posible proponer una nueva situación, según surgen de la comparación entre estructuras pertenecientes a lo ya conocido y a los nuevos productos culturales. Es un debate, por tanto, entre el pasado y el presente, condicionado por el contexto cultural en el que los lectores se encuentran en sí mismos.

Así, nos proponemos con este trabajo concretar y materializar algunas cuestiones que han protagonizado y protagonizan el debate en torno a la literatura digital, esto es, aquella que se escribe para ser reproducida exclusivamente en los nuevos medios digitales. Categorías como las de ‘autor’ y ‘lector' serán analizadas bajo este nuevo prisma a propósito de la obra que hemos escogido, Condiciones Extremas, así como la perspectiva lúdica de buena parte de las propuestas literarias que podemos hoy encontrar en la red. 


\section{2 - Literatrónica. una cuestión terminológica}

Literatrónica, que es el nombre del proyecto que comentamos, y en donde se alojan distintas obras que intenta materializar las características que allí se defienden, se no presenta como Narrativa Digital Adaptativa.

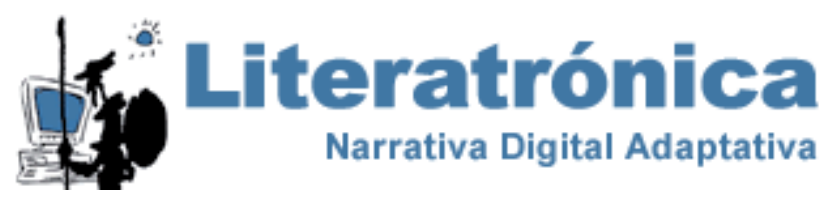

Parece que el autor quisiera que quedara perfectamente claro a lo que nos enfrentamos cuando decidimos dedicar nuestro tiempo a la lectura de alguna de las obras propuestas.

Las definiciones de lo literario en el contexto digital, de la literatura digital, digamos, han sido numerosas a lo largo de las últimas décadas. Dice Laura Borrás (2005: 4I) que la teoría de la literatura debe identificar y describir las nuevas formas de textualidad electrónica, porque aún no está claro si trata, en todos los casos, de literatura. En principio, estas novedosas manifestaciones se recogen bajo denominaciones como las que, a continuación, vamos a observar:

- Literatura digital frente a literatura impresa: el texto impreso es estático y el digital, dinámico.

- Literatura electrónica: nacida de y para el espacio digital, el ordenador, es decir, que no puede ser impresa, ni leerse fuera de este medio.

- Ciberliteratura: término que nos sitúa de manera amplia en el ámbito de la literatura generada por ordenadores, propuesta literaria donde la máquina puede ser considerada como co-autora del texto. Sin embargo, no todos los textos digitales tienen por qué ser cibertextos, en la medida en que pueden ser únicamente traducciones en formato papel traducido a la pantalla.

- Literatura hipertextual: funciona en base a una estructura de hipertextos, es decir, a partir de la interconexión de fragmentos, en principio, textuales. Algunos autores hablan de proto - hipertextos, como los antecedentes impresos en papel de los hipertextos electrónicos, como Rayuela o Ulises.

- Hiperliteratura: Término derivado de hipertexto, hace referencia a la literatura multilineal surgida de la lectura de sus lectores.

- Literatura ergódica (Aarseth, 2004: II7-II8): tipo de literatura que espera y reclama un esfuerzo, más allá de pasar páginas, por parte del lector. Aarseth ha acuñado el término a partir de los vocablos griegos ergon y hodos, que significan 'obra' y 'camino', y lo usa para referirse a las creaciones literarias que exigen un esfuerzo nada trivial que permita al lector atravesar el texto. Ello nos remite a un universo literario no exclusivamente digital, sino que también abarca la producción en papel, como se ve en el caso de Rayuela, de Cortázar. 
Estas son algunas de las etiquetas más utilizadas dentro del marco de las investigaciones en España. No obstante, Laura Borrás recoge también en su estudio introductorio denominaciones como Multicurse literature, Blended genre, Web texts, que requieren un mayor espacio del que podemos ofrecer aquí. A ella remitimos, por tanto.

Sin embargo, Literatrónica se nos presenta de otra manera, no sólo en cuanto a su "etiquetado", sino también en lo concerniente a su contenido. Es una forma de escritura y lectura en la que los vínculos en el texto llevan al lector a distintos destinos dependiendo de lo que éste haya leído antes. Una obra literaria adaptativa se reconfigura para el lector produciendo cada vez una experiencia única de lectura. En otras palabras, el medio actúa en el mensaje. La adaptatividad puede ser compleja, como en el caso de Condiciones Extremas, una obra que se presta para lectura no-lineal gracias a un arreglo circular de eventos, o simple como en el caso de Elprimer Vuelo de los Hermanos Wright, una obra diseñada para ser leída en forma más o menos lineal.

Uno de los principales argumentos que los oponentes del hipertexto literario han esgrimido en el pasado es la historia fragmentada que se le ofrece al lector. En este caso, el lector recibe una trama que ha sido optimizada desde el punto de vista narrativo. Es decir, el lector recibe un texto lineal la mayor parte del tiempo, favoreciendo no sólo la esencia del medio digital, sino la esencia de la narrativa en un sentido clásico: inmersión. Así, un texto adaptativo no podrá ser reproducido en papel salvo, quizás, como una ruta de lectura en un momento dado. Por tanto, en Literatrónica estamos ante textos que no pueden existir sin el medio electrónico.

En Literatrónica, pues, se aúnan propiedades que en otras definiciones funcionan como núcleo de las mismas. Podríamos decir que estamos ante un tipo de texto ergódico, a la manera de Aarseth, desde el momento en que tenemos que decidir qué itinerario de los propuestos queremos seguir; que avanzar de un texto a otro requiere de enlaces y nodos, es decir, que jugamos con hipertextos; que si el medio electrónico o digital es absolutamente imprescindible, estamos ante literatura electrónica o digital; que por el entorno en el que se desarrollan estas obras, el ciberespacio, podemos considerarlas ciberliteratura. Se podrían hacer muchas matizaciones, pero lo que aquí nos interesa es señalar que Literatrónica no rompe con otro tipo de obras, de proyectos, sino que une en sí misma, tal vez, lo que más beneficioso de unos y otros para la lectura de los usuarios. En definitiva, será esa capacidad adaptativa la que se convierta en el punto diferencial con respecto a otras experiencias, puesto que el objetivo es que en ningún momento el lector pueda sentirse perdido, angustiado por el exceso de enlaces, aburrido o desorientado en un marasmo de caminos, de saltos hacia atrás y hacia adelante, que como experimentos literarios o artísticos son muy apreciados, pero que como lectura son desesperantes. 
CONDICIONES EXTREMAS, de Juan B. Gutiérrez

\section{I - Línea argumental}

Condiciones Extremas es una obra de narrativa digital que ha sufrido tres procesos de re-escritura con tres técnicas diferentes. La que aquí comentamos, fue construida entre 2003 y 2005. Esta versión (2005) fue esencial en la construcción del motor especializado de inteligencia artificial que funciona en Literatronica.com. Esta obra es un experimento sobre lectura no-lineal en el medio digital, ya que el arreglo circular de eventos permite naturalmente saltos narrativos. Hemos hablado de ello más arriba.

Básicamente, el lector se encuentra ante una obra de ciencia ficción cuya historia se desarrolla en Santafé de Bogotá en tres momentos: 1998, 2050 y 209O. El industrial Índigo Cavalera, la científica Miranda Macedonia y el mutante Equinoccio Deunamor. Son los tres personajes principales, presentes en los tres momentos, en cada uno con una misión, sabiendo quizás algo que en otro momento ignoran, no necesariamente siguiendo la lógica temporal. La aparición de la raza mutante, la contaminación del planeta y la invención de la máquina del tiempo son algunos de los temas que estructuran la obra. Como dice Susana Pajares (I998): "Hay intriga, persecuciones, búsquedas, secuestros, enigmas... en un movimiento constante de la acción narrativa. Pero Condiciones Extremas no se reduce a esto, pues afloran cuestiones de peso tras la vestidura de la cienciaficción: el poder, la ambición, la organización social, el racismo, el miedo, y ciertamente una reflexión crítica sobre los extremos a los que puede llegar el nivel de contaminación de nuestro planeta".

A lo largo de toda la obra el autor intercala partes narradas con diálogos concisos, y todos los fragmentos o capítulos del texto se van relacionando a través de los enlaces que aparecen al final de cada uno de ellos, tres para ser más concretos, ordenados según la relevancia narrativa. En ningún caso el salto de un texto a otro provoca sensación de pérdida. El lector avanza en su lectura eligiendo, sí, pero con la seguridad de que, sea su elección la que sea, habrá recorrido un itinerario bien dibujado, coherente, hasta llegar a un final. La continuidad narrativa, a pesar del salto, está asegurada.

Pajares Tosca (1998) nos motiva a experimentar la sensación de una lectura multilineal como ésta: "La clave de la estructuración de este hipertexto es el juego con el tiempo, pues las diferentes épocas se superponen y enlazan de un modo que la simple lectura lineal no podría mostrar. Al lector se le antojaran relaciones inesperadas porque en el fondo continuamos preparados para leer linealmente, y cuando la secuencia temporal lógica es desplazada por otro tipo de asociaciones (temáticas, connotativas, evocadoras, etc.), no sólo nos desconcertamos, sino que nos damos cuenta de los mecanismos que rigen nuestra forma de leer precisamente porque están ausentes. Y ésa es una experiencia muy sana que nadie debería perderse".

La estructura de Condiciones Extremas es arborescente. Una vez que escogemos una rama, no podemos dar marcha atrás si queremos llegar a buen puerto (es cierto que 
en la mayoría de las páginas web siempre se puede “ir atrás”, sin embargo hacerlo aquí supondrá deshacer la continuidad narrativa y volver a empezar, tomar un camino totalmente distinto). Al mantenerse cada rama estrictamente aislada de las demás, estos textos con forma arborescente controlan el itinerario del lector desde la raíz hasta el extremo de la hoja y ofrecen indudables garantías de que de las elecciones resultará siempre una historia perfectamente formada. Esta es la estructura típica de los libros juveniles de" Elige tu propia aventura".

\section{2 - Autor/Lector}

Sobre las reconfiguraciones de las categorías de autor y de lector se ha escrito mucho. Desde la muerte del autor hasta el autolector, la bibliografía no ha hecho más que crecer. En el caso de Literatrónica habremos de hacer algunas aclaraciones al respecto. María Elena Barroso Villar (2005: 74-90) resume claramente las nuevas concepciones de estas dos figuras y así explica que los lectores de hipertextos tienen libertad para decidir el itinerario de su lectura y, en consecuencia, el acto de leer abandona la linealidad secuencial de la lectura de libros impresos, por eso el modelo hipermedia requiere una interactividad, una capacidad de quien la usa para ejecutar el sistema, estableciéndose diferencias sustantivas entre lector y usuario de hipertextos. Para Barroso Villas, con quien no coincidimos en este aspecto, ese usuario pasaría a convertirse en coautor del texto, no ya sólo en cuanto a la construcción de los sentidos, sino como artífice de su arquitectura. El papel del lector, dice María Jesús Orozco Vera (2005: 97), “asume un notable protagonismo". Recordemos también cómo Barthes o Foucault diluían la figura del autor en pos del engrandecimiento de la del lector.

Sobre esa libertad del lector también hablan Douglas (I992) y Bolter (I99I), y a partir de ellos la gran mayoría de los estudiosos de la literatura electrónica. Pero tener la posibilidad de elegir entre una serie de nodos es bastante diferente que ser el autor de un texto, es decir, igualar esos procesos, el de la lectura y la escritura, es aquí imposible. Como afirma Pajares Tosca (2004: 38), "se podría decir que las elecciones de un lector de hipertexto resultan paradójicamente reductoras, al restringir a un solo camino de lectura lo que en principio ofrecía varias posibilidades."

Para Stalin (I990: 877), el hipertexto reproduce el modo de procesar de la mente humana. Sin embargo, los enlaces están predeterminados por un autor y sólo indican un desarrollo del texto.

Hay, sin embargo, un matiz que nos gustaría señalar aquí y ahora, y que puede funcionar como puente entre posturas contrarias. Es cierto que convertir al lector en autor es una exageración, si por escritura entendemos una actividad que requiere imaginación y habilidades lingüísticas muy complejas. Aún así, podríamos afirmar que el lector de las narraciones con elecciones múltiples, como dice Marie-Laure Ryan (2004: 340), "se convierte en autor, no en el sentido creativo del término, sino porque ejerce una au- 
toridad sobre los personajes". Así, el lector de Condiciones Extremas ciertamente podría considerarse coautor desde esta perspectiva, que nos parece equilibrada.

Lo que sí que hace el lector es actuar. Este término parece más adecuado que el de interactividad, que no responde a la realidad puesto que en todo proceso interactivo los participantes poseen las mismas posibilidades a la hora de emitir y recibir señales, cosa que no ocurre en el hipertexto, en el que, como vemos, el lector no está en el mismo nivel que el autor, por mucho que pueda escoger entre lo que el autor ofrece, como ocurre en la obra Condiciones Extremas, en Literatrónica. El lector, pues, actúa, navega a través de una estructura predeterminada. Esa navegación se realiza mediante saltos de enlace a enlace, y esta es una de las dificultades que podrían perjudicar el proceso lector de hipertextos, ya que, si no existieran suficientes indicios para la orientación del lector, cada enlace podría resultar para éste una puerta hacia algo desconocido que dificultaría la comprensión. Depende, por tanto, y es lo que ocurre en Literatrónica, de la capacidad creativa y organizativa del autor que el lector no acabe perdido y vagando de enlace a enlace en busca de una salida. En nuestro caso hay enlaces, hay itinerarios, pero están perfectamente diseñados con el único objetivo de no desorientar.

Con esto queda claro que el hipertexto no significa la muerte del autor, es más, el autor está más presente al concebir su obra como una idea múltiple. De la misma forma, no podemos llamar a los lectores "coautores" cuando su actividad se reduce a decir un itinerario u otro. Evidentemente podríamos llamarlos así, pero entonces necesitaríamos de otras etiquetas o designaciones para referirnos a quienes a lo largo de toda la historia de la humanidad han elaborad por sí mismo una obra de arte, es decir, a los autores.

El modelo hipertextual o hipermediático requiere la capacidad de quien lo usa para ejecutar un sistema, para participar activamente. Esta acción supone diferenciar entre lo que se ha entendido habitualmente como receptor y lo que en los últimos años se denomina 'usuario'. Uno de los teóricos que profundizó y puntualizó el concepto fue Espen Aarseth (2004: II8-II9) y así "la actuación del lector se produce enteramente en su cabeza, mientras que el usuario del cibertexto también actúa en un sentido extranoemático. Durante el proceso cibertextual, el usuario habrá efectuado una secuencia semiótica, y este movimiento selectivo es una labor de construcción física que no se describe en los distintos conceptos de "lectura". Este fenómeno lo denomino "ergódico", sirviéndome de un término apropiado de la física, que deriva de las palabras griegas ergon y hodos, las cuales significan respectivamente "trabajo" y "camino". En la literatura ergódica, es necesario un esfuerzo no trivial que permita al lector transitar por el texto, como ya hemos apuntado a la hora de proponer definiciones.

Estos textos, en consecuencia, lo que requieren son un tipo distinto de lectura, y a este respecto David Riesman (I950: 259) señaló la diferencia que existía entre el lector centrípeto y el lector centrífugo. El primero se distingue por su habilidad para definir su papel como lector a medida que descubre nuevas formas de orientarse en el espacio narrativo o mediante la revisión del concepto de desenlace y cierre. El lector centrífugo, por el contrario, orienta su lectura a partir de sus conocimientos sobre la práctica lectora 
tradicional y sobre las convenciones literarias, lo que les lleva a encontrar frustrantes o desprovistos de sentido relatos que se apartan de las normas.

Esta narrativa digital, como indica Yellowlees Douglas (2003: I80), parece exigirnos que nos transformemos en lectores centrípetos, que van más allá de la simple percepción del texto virtual de un autor y que nos rebelemos ante las prescripciones autoriales para obtener nuestras propias lecturas. En nuestro caso, en la obra Condiciones Extremas, vamos decidiendo por cada página leída entre tres opciones, según la mayor o menor relevancia que puedan tener para nuestra lectura. Por lo tanto, también aquí, avanzar en nuestra lectura supone decidir, actuar, participar de lo que un autor nos ofrece.

Una obra literaria adaptativa como la que aquí estamos comentando seguiría, para sintetizar, un esquema como el que vemos en la ilustración seguidamente. Entre el autor y el lector el sistema de información va generando, según las elecciones del segundo y las posibilidades narrativas del texto, itinerarios posibles de lectura. A la vez, va informando al autor de dichas elecciones para que éste pueda analizar el éxito o el fracaso de la experiencia lectora de su interlocutor. Al elegir el lector el hipervínculo, o uno de ellos, automáticamente es guardado en la hoja de ruta del sistema de información, que a continuación calcula qué páginas pueden resultar más interesantes para esa experiencia de lectura y así se las ofrece al usuario-lector. Por lo tanto, podemos decir que lo importante de toda esta estructura textual es el poder garantizar una experiencia de lectura satisfactoria. El sistema, pues, sugiere continuamente caminos. Y he aquí una de las novedades más significativas. Ese sistema de información sí está funcionando como si de un "autor digital” se tratase. Elige y decide según la lógica textual. Hay un diseño previo pero es el ordenador el que, en función de la narratividad, decide qué páginas ofrecer. 


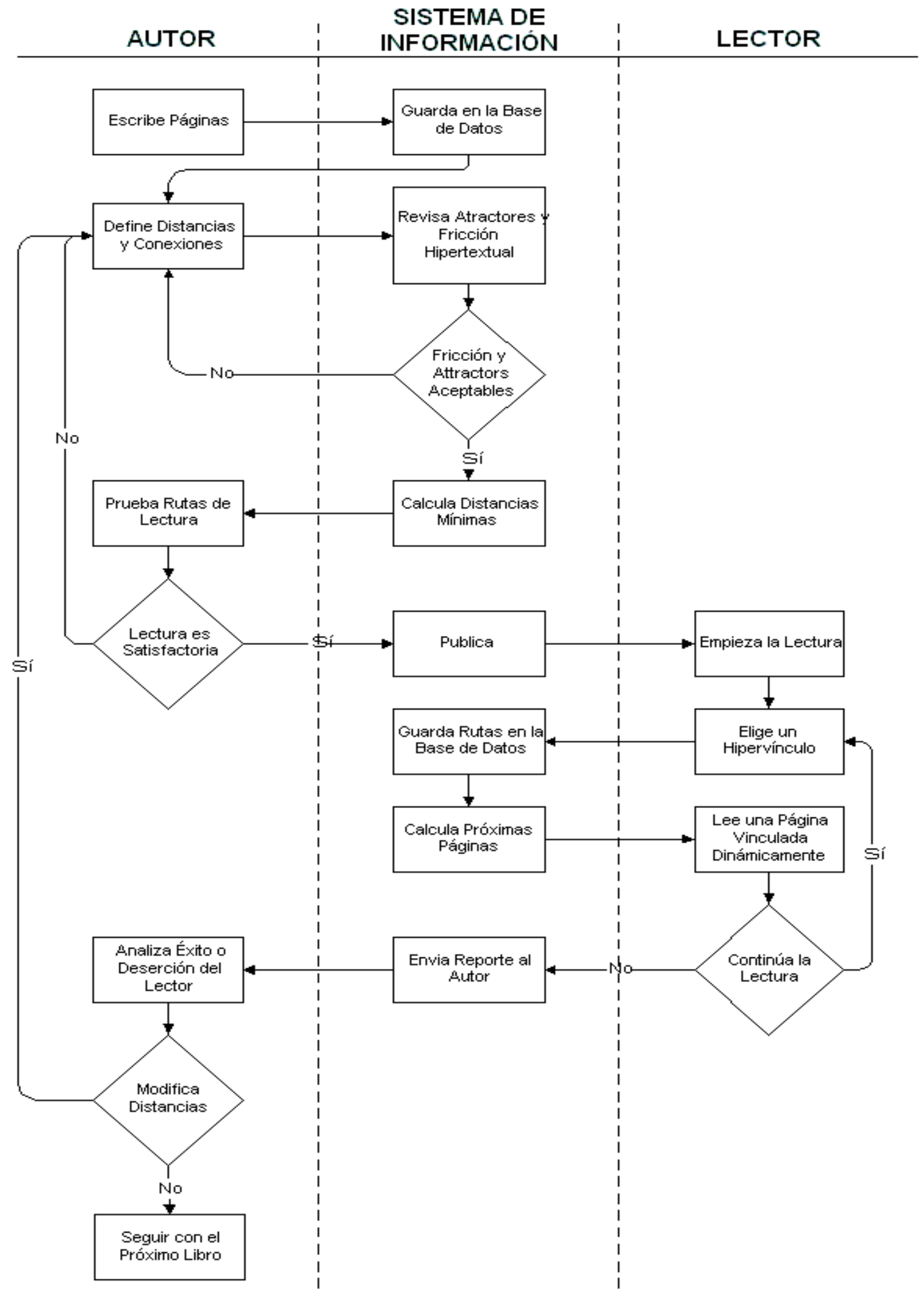


De la misma manera, en sintonía con lo anterior, existe en cada libro un mapa de lectura en el que se nos informa del porcentaje de la obra que ya está leído y todo lo que nos queda por leer. Sabemos así que no habrá textos “perdidos”, esto es, fragmentos que aparezcan en una lectura y no en otra y que no sepamos cómo volver a ellos. En este mapa están todos los textos posibles, si bien no hay combinación de los mismos, es decir, si decidimos leer Condiciones Extremas, nuestro itinerario no seguirá el orden de este mapa, puesto que ya entrará en juego la combinatoria o esa "capacidad adaptativa” de $\mathrm{Li}$ teratrónica. En la primera de las dos ilustraciones siguientes podemos ver cómo se pude acceder a ese mapa clicando sobre "Mapa", y en la segunda, la disposición de los textos numerados y por orden.

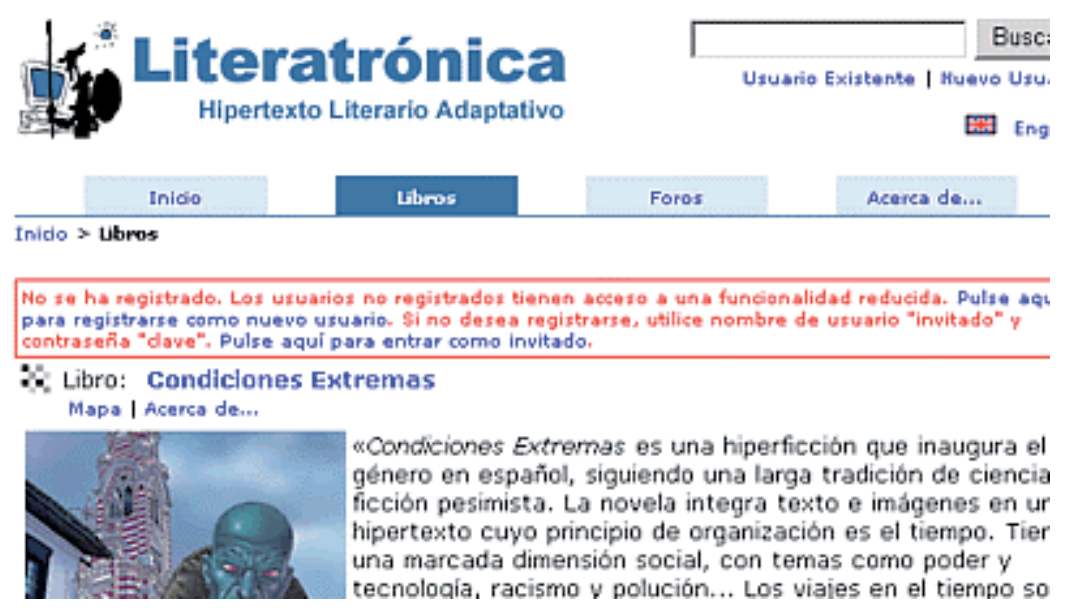
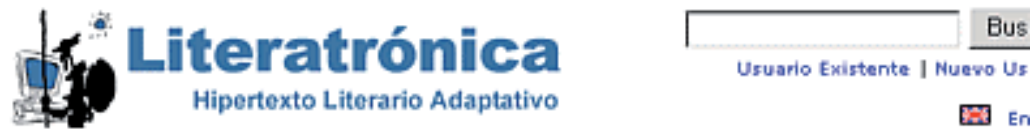

En En

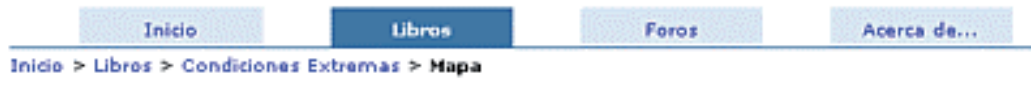

Ho se ha registrado. Los usuarios no registrados tienen acceso a una funcionalidad reducids. Pulse ac para registrarse como nuevo usuario. si no desea registrarse, utilice nombre de usuario "invitado" y contraseña "clave". Pulse aquí para entrar como invitado.

W Libro: Condiciones Extremas

Mapa I Acerca de...

1 Décima Década

El descubrimiento de un Avatar derivado del género humano no fue el único íncider que removió la historia de la segunda mitad del siglo. Un hecho más se produjo; de este, sin embargo, pocos tuvieron noticia, y cada uno de los involucrados fue...

2 Dêcima Década

Miranda detuvo su automóvil cerca a una estación de servicio. Tenía aún combust nara rientos de vilhmetros nern le interecaha nanar tiemnn antes de llenar al rent 


\section{3 - Bifurcación de itinerarios. El texto como juego}

La posibilidad de la elección en un texto por parte del lector no es nueva, ni lo fue con la llegada del hipertexto. En sus Escrituras nómades, Belén Gache ya recogía un compendio de obras que a lo largo de la historia de la literatura universal hacían gala no sólo de esa elección, sino también de otras propiedades como la discontinuidad, la multilinealidad, etc. De la misma manera, el componente lúdico de ciertas obras centenarias en edad ponía de manifiesto que ni tan siquiera las nuevas tecnologías aplicadas a la literatura resultaban novedosas a este respecto. Lo que sí que ha resultado definitivo ha sido las enormes posibilidades que el medio digital ofrece para combinar propiedades como las que acabamos de señalar. El ordenador, por tanto, se configura como medio específico y poderoso capaz de unir, condensar o intercalar distintas opciones a la vez.

Una de las propiedades que más se han señalado a raíz de la popularización del hipertexto es la de la bifurcación de itinerarios, es decir, la de la posibilidad de optar por caminos distintos que ofrecen experiencias de lectura también distintas, como materialización de teorías literarias ensayadas por Borges, Calvino o Cortázar. Tal vez sea el relato Eljardín de senderos que se bifurcan el texto fundacional o más significativo a este respecto, metáfora por entonces de un posible texto infinito que se iría diversificando sin encontrar fin alguno. La idea central del cuento "El jardín...”, nos dice Calvino (I997 a) es la de un tiempo múltiple y ramificado en el que todo presente se bifurca en dos futuros, de manera que forman una red creciente y vertiginosa de tiempos divergentes, convergentes y paralelos.

De la misma manera, la novela de Calvino Si una noche de invierno un viajero... ponía sobre la mesa la posibilidad de la multiplicidad, con el propósito de dar la esencia de lo novelesco concentrándola en diez comienzos de novelas, que desarrollan de las maneras más diferentes un núcleo común y que actúan en un marco que los determina y está a su vez determinado por ellos.

La publicación de Rayuela, de Julio Cortázar, abre definitivamente las puertas a un tipo de lectura diferente. Ofrece dos posibilidades de lectura, una lineal y otra multilineal o discontinua en la que el lector puede seguir un esquema diferente al clásico de capítulos ordenados numéricamente. Esta segunda vía de lectura no surge sólo como itinerario lúdico (la rayuela es un juego muy antiguo), sino también como búsqueda, y no olvidemos que el propio Cortázar quiso titularla Mandala, que es precisamente la metáfora gráfica de una búsqueda, un viaje hacia el interior del yo, como nos dice Alazraki (r994). La estructura de Rayuela como un juego de piezas movibles y rearmables responde a una concepción de la novela como un orden abierto y una combinatoria en la que cada lector escogerá el libro que ha elegido leer. Esa estructura aleatoria supone una transgresión que centra la novela menos en los personajes fabulados que en ese personaje - cómplice que se busca confabular. Novela-puente entre el autor y el lector en la que el primero invita al segundo a entrar en el juego y a ejercer su derecho de participación por medio de su propia combinación, técnica también de los mandalas. 
Podríamos señalar otros hitos importantes, y sin embargo hemos querido hacer hincapié en estas tres obras porque nos ayudan a entender la construcción y el desarrollo de Condiciones Extremas, en Literatrónica. En primer lugar, es evidente que la bifurcación de itinerarios es uno de los puntos álgidos de la obra. Por razones de combinatoria numérica, cada página leída ofrece tres posibilidades de continuación para el lector. Nos sentimos como si estuviéramos eligiendo nuestra propia aventura, a la manera de los ya clásicos textos de los años ochenta que tanto éxito cosecharon y a tantos lectores dejaron atrapados. Nuestra lectura se bifurca en un momento determinado. ¿Qué camino elegir? Podríamos guiarnos por los porcentajes que más o menos nos indican la opción que mejor se ajustaría a nuestra experiencia lectora. Pero... ¿y si eligiéramos otra? Sin embargo, no podemos obviar que en Condiciones Extremas existe un control del guión narrativo previo que impone límites a la libertad de movimiento, de tal manera que elijamos la opción que elijamos, todos los recorridos de nuestra lectura constituyen una historia coherente. En segundo lugar, la opción del "Mapa" nos da la posibilidad de empezar a leer en el capítulo que queramos, y además nos deja saltar a otro sin necesidad de seguir ningún camino. La multiplicidad de lectura es también aquí significativa. Podríamos empezar por el capítulo que quisiéramos, a la manera calviniana, aunque en este caso perderíamos el hilo argumental con facilidad. Por último, si Rayuela nos ofrecía dos opciones de lectura tan diferentes, igualmente en Condiciones Extremas podemos decantarnos por seguir con una lectura lineal, sin preocuparnos por elecciones o itinerarios, o bien optar por esquemas bien distintos, dejándonos guiar a través de múltiples caminos. Tan sólo es necesario decidirse, arriesgarse. Jugar.

El concepto de juego es uno de los más relevantes del pensamiento del siglo XX. La lista de sus manifestaciones cubre una enorme variedad de trabajos y fenómenos que van desde el estudio Homo Ludens de Johan Huizinga, la taxonomía de los juegos de Roger Caillois, la noción de juegos del lenguaje de Wittgenstein, la filosofía del "como si" de Hans Vaihinger, la semántica de la teoría de los juegos de Jaakho Hintikka, la doctrina del juego de los signos de Derrida o la fascinación por la combinatoria de Nabokov y Calvino, o el movimiento Oulipo, entre otras, todas hasta llegar al advenimiento de la cultura informática y los juegos electrónicos. Entre los postmodernos el juego está especialmente bien considerado porque ejemplifica el carácter elusivo del significado y la naturaleza escurridiza del lenguaje.

Roger Callois (I96I) distinguió en su día cuatro tipos de juegos que configuran una tipología valiosísima para poder establecer similitudes entre los textos y los propios juegos:

- Agon: juegos en los que lo esencial es competir (fútbol, tenis).

- Alea: juegos de azar, como la ruleta y la lotería.

- Mimicry: juegos de imitación.

- Ilinx: juegos que suponen metamorfosis, inversión de categorías establecidas, como los bailes de disfraces. 
La primera de estas categorías, agon, tiene una utilidad muy restringida en el campo literario. Por esa especie de competición, tal vez en la ficción hipertextual encontremos un buen ejemplo, por ese antagonismo entre lector y texto. Al acercarse al texto como si fuera un juego de ordenador, algunos lectores tienen la impresión de encerrarse en un laberinto lleno de caminos secretos diseñados por el autor. Pero podríamos también definir el hipertexto como un tipo de agon si lo consideramos un puzle que hay que resolver. De una forma o de otra, la ficción hipertextual se construye en muchas ocasiones en torno a la noción de texto como juego, o al menos éste es uno de los factores más determinantes. Dependerá del autor el que dicho juego pueda o no resolverse, pueda o no satisfacer a quienes jueguen con él. En Condiciones Extremas sabemos que la lectura necesita de nuestra participación, pero a diferencia de otras experiencias hipertextuales, aquí se nos advierte desde un principio que llegaremos a buen puerto, que no nos perderemos. Resolveremos, pues, el puzle. De distintas maneras, sí, pero resuelto en todas.

La idea de convertir el texto en alea, esto es, en un juego de azar, afecta igualmente a nuestro análisis de Condiciones Extremas. Sabemos que Oulipo tenía predilección por la combinatoria. Italo Calvino, uno de sus miembros, define la literatura como juego combinatorio que juega con las posibilidades intrínsecas del propio material. Este mismo principio es el que pone en práctica Cent mille milliards de poèmes, de Raymond Queneau, una colección de sonetos impresos en páginas cortadas en tiras, de manera que moviendo las tiras y combinando los versos, el lector puede obtener una elevadísima cantidad de textos diferentes. Recordemos que la obra que comentamos se fundamenta en un sistema de combinación narrativa llevado a cabo por el ordenador. El propio Juan B. Gutiérrez, autor de la obray del portal Literatrónica, afirma que el concepto más cercano de esta obra y de este proyecto es el hipertexto escultórico, en el que todas las páginas están conectadas y el autor elimina los enlaces. La diferencia aquí, sin embargo, es que es el equipo el que crea de forma dinámica las conexiones.

Las dos últimas categorías, mimicry e ilinx, también podemos relacionarlas con las ficciones hipertextuales y con toda la literatura digital. En el primer caso, porque todo lo que tenga que ver con la imitación estaría relacionado con cuestiones inmersivas e interactivas. En Condiciones Extremas, ya lo hemos dicho, se respetan ambas, es decir, el sistema de información de Literatrónica está configurado para que los textos que se ofrezcan al lector sean los más adecuados inmersivamente, que respeten la narratividad y la causalidad, pero a la vez necesita de la acción del lector-usuario, que es el que debe pulsar con el ratón sobre la opción que elija. Con el ilinx, lo sabemos, abandonamos el reino de las actividades gobernadas por reglas y entramos en el terreno del juego libre. El literatura el juego libre está relacionado con lo que Bajtín llamó "lo carnavalesco", esto es, la desestabilización de todas las estructuras, incluidas las que crea el propio texto. No es el caso de la obra que comentamos, sin embargo sí que dentro de esta desestabilización podríamos situar teorías como la de la deconstrucción, la muerte del autor, el rizoma, etc., en donde saltan por los aires jerarquías, categorías, significados y entidades. 
Sobre todo nos interesa señalar cómo la crisis de la idea de representación ha favorecido la metáfora del juego para expresar las preocupaciones del pensamiento posmoderno. Esta crisis se debe en gran parte a la influencia de la lingüística saussureana, especialmente a su insistencia en el carácter arbitrario del signo lingüístico, no mimético. De ahí, la literatura puede considerarse un juego porque como cualquier tipo de discurso especializado, está gobernada por sus propias convenciones arbitrarias. Si la literatura es un juego se debe a que el lenguaje también lo es, y si el lenguaje es un juego, es porque sus reglas forman un sistema autoinclusivo que determina nuestra manera de captar la realidad.

Fue el paso del estructuralismo a la deconstrucción el que desplazó el foco de interés de las metáforas lúdicas del ludus a la paidia, es decir, de la noción de juego como actividad gobernada por reglas a la de juego como subversión de las reglas.

En la actualidad, ya centrados en el hipertexto, comprobamos que, de un primer momento en el que predominó la experimentación, estamos en otro muy distinto en el que vuelve a ser fundamental la capacidad de los autores para contar una historia. Los diseñadores de textos interactivos que están hoy trabajando para el sector comercial tienden a adoptar una visión de la narratividad mucho más estrecha y clásica que muchos escritores, digamos, académicos. Saben que el éxito popular de una obra interactiva depende de su habilidad para crear una experiencia inmersiva, y las estructuras narrativas clásicas han demostrado a lo largo del tiempo ser la receta más adecuada para mantener la atención del usuario.

\section{3-Conclusiones}

Si algo hemos querido destacar y defender a lo largo de nuestro análisis ha sido el hecho de que Condiciones Extremas apuesta por la continuidad narrativa por encima de cualquier otro aspecto. Está escrita para ser leída en medios electrónicos, usa hipervínculos en su desarrollo y necesita de la participación activa de los lectores, cualidades que han sido señaladas por muchos como las culpables de la desorientación de quienes leen hipertextos y de la sensación de agotamiento. Se tome el itinerario que se tome, los lectores llegarán a cada uno de los finales de una manera satisfactoria. En un texto lineal, cuanto más leemos y más sabemos sobre el mundo textual, mayor capacidad tenemos para poder anticiparnos a los acontecimientos. La continuidad de la línea argumental funciona a la manera del hilo de Ariadna, esto es, el lector va atando retazos de información que mantiene unidos para poder acceder a ellos más fácilmente, como nos dice Ryan (2004: 3II). Sin embargo, la estructura rota del texto interactivo, en este caso hipertextual, priva a la memoria de uno de los métodos de almacenamiento más eficientes. En Condiciones Extremas, ya lo dijimos, esta estructura no sólo no perjudica la experiencia lectora, sino que la potencia gracias a su sistema de información. He aquí un ejemplo de texto electrónico y perfectamente inmersivo. 
La sensación de pérdida y de deambular sin rumbo fijo, propias de la progresión del lector a través de una cantidad elevada de hipertextos, desaparece nada más comenzar nuestra lectura. El sistema nos guía, el autor real y el "autor digital” funcionan como una doble brújula que garantiza la narratividad del texto. Si un sistema de enlaces suele tentarnos a movernos continuamente, sin permanecer demasiado en aquel no-lugar al que accedemos, el que nos propone Condiciones Extremas nos limita el margen de movilidad para hacer nuestra experiencia más gratificante.

Seguridad y significación están aquí garantizadas. No ha sido siempre así. Los primeros autores de hipertextos, condicionados unos por la teoría, radicalizados otros por la vanguardia, se decantaron por privar al público de toda sensación de seguridad en vez de ir atrayéndolo poco a poco a los nuevos hábitos de lectura. De la misma manera, el modelo de la novela creó un tipo de expectación que subordinaba el significado local a la estructura narrativa global, con toda la desorientación que ello suponía a lectores no acostumbrados a moverse en este tipo de textualidades.

Es posible, por tanto, conjugar, como dice Ryan, la interactividad y la inmersión. Es posible disfrutar de la lectura de una hiperficción sin riesgo de desesperarse, agotarse o rendirse. Es lo que hemos querido defender a lo largo de todo este análisis. Es posible, finalmente, que Condiciones Extremas se convierta en el eslabón entre dos maneras muy diferentes de leer, una clásica, milenaria casi, y otra novísima que con el paso de los años irá afianzándose más y más y ganando adeptos entre las nuevas generaciones. 


\section{Referencias}

-Aarseth, E. (2004). “La literatura ergódica”. En Literatura y Cibercultura, Domingo Sánchez Mesa (ed.), п8-I45. Madrid: Arco/Libros.

- Aguirre Romero, J. Ma. (2OII). "La transformación de la sociedad lectora con el impacto tecnológico: el futuro lector". En Literatura e Internet. Nuevos textos, nuevos lectores, Salvador Montesa (dir.), págs. 19-4O. Málaga: AEDILE.

-Alazraki, J. (1994). Hacia Cortázar: aproximaciones a su obra. Barcelona: Antropos Contemporáneos.

- Baetens, J. (2003). Close Reading Hyperfiction. Leuven/Maastricht: University of Leuven/ University of Maastricht (mimeo).

- Barroso Villar, M. E. (2005). "La comunicación literaria en el nuevo universo tecnológico". En Comunicación, universo artístico y nuevas tecnologías, María Elena Barroso Villar (ed.), 5793. Sevilla: Alfar.

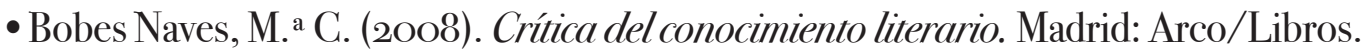

- Bolter, J.D. (199I). Writing Space: The Computer, Hypertext and the History of Writing. New Jersey: Lawrence Erlbaum Associates.

- Borges, J.L. (1997). Eljardín de senderos que se bifurcan. Madrid: Alianza.

- Borrás Castanyer, L. (ed.) (2005). Textualidades electrónicas. Nuevos escenarios para la literatura. Barcelona: Editorial UOC.

-_____(2OII). "Nuevos lectores, nuevos modos de lectura en la era digital”. En Literatura e Internet. Nuevos textos, nuevos lectores, Salvador Montesa (dir.), págs. 4I-66. Málaga: AEDILE.

- Callois, R. (ig6i). Men, Play, and Games. Nueva York: Free Press.

- Calvino, I. (1997a). Seis propuesta para el próximo milenio. Madrid: Siruela.

•_____ (1997b). Si una noche de invierno un viajero. Madrid: Siruela.

- Cassany, D. (2012a). "La metamorfosis digital: cambios, ventajas y riesgos de leer y escribir en la red". En Las TIC en la escuela, nuevas herramientas para viejos y nuevos problemas, Daniel Goldin, Marina Kriscautzky y Flora Perelman, págs. 2I7-236. Barcelona: Océano.

•_____(2012b).En_línea. Leery escribir en la red. Barcelona: Anagrama. 
- Chico Rico, F. (2003). "Retórica e hiperficción”. En Escrituras digitales: Tecnologías de la creación en la era virtual, Virgilio Tortosa (ed.), 99-I20. Alicante: Universidad de Alicante.

- Cortázar, J. (1967). Rayuela. Buenos Aires: Sudamericana.

- Douglas, J.Y. (1992). Print Pathways and Interactive Labyrinths: How Hypertext Narratives Affect the Act of Reading. New York: New York University.

-Echeverría, J. (2003). “Cuerpo electrónico e identidad”. En Arte, cuerpo, tecnología, Domingo Hernández Sánchez (ed.), I3-29. Salamanca: Ediciones Universidad de Salamanca.

- Gache, B. (2006). Escrituras nómades. Gijón: Trea.

- Goicoechea, M. a (2007). "Hyper-Paradigm". En Literatures in the Digital Age: Theory and Praxis, Amelia Sanz and Dolores Romero (eds.), I8-2r. Newcastle: Cambridge Scholars Publishing.

- Gutiérrez, J. B.(2005). Condiciones Extremas, http://www.literatronica.com/src/Pagina.as px?lng $=$ HISPANIA\&opus $=$ I\&pagina $=\mathrm{I}$

-____(2003). Literatrónica. Sobre cómo y por quécrearficción para medios digitales, http:// www.cibersociedad.net/congreso/comms/co4gutierrez.htm

- Jenkins, H. (2008). Convergence Culture: La cultura de la convergencia de los medios de comunicación. Barcelona: Paidós.

- Landow, G. (2008). Hipertexto 3.o. Teoría crítica y nuevos medios en la era de la globalización, Barcelona: Paidós.

- Marino, M. (2006). Literatronica. An interview with creator Juan B. Gutierrez, http:/ / writerresponsetheory.org/wordpress/2006/o8/29/literatronica-an-interview-with-creator-juanb-gutierrez

- Mendoza, A. (2010a). "La lectura del hipertexto literario. El despliegue de referentes, conexiones e hipervínculos en la formación del lector”. En El lector ante la obra hipertextual, A. Mendoza y C. Romea (Coords.), págs. I43-I74. Barcelona: Horsori.

•____ (20Iob). "Canon e hipertexto”. En El invisible anillo, ı2, págs. 32-45.

-_____ (2012a). "Presentación". En Leer hipertextos. Del marco hipertextual a la formación del lector literario, Antonio Mendoza (coord.), págs. 9-32. Barcelona: Octaedro. 
-____(2OI2b). "Leer hipertextos de papel: sobre el lector y sus hipervínculos cognitivos". En Leer hipertextos. Del marco hipertextual a la formación del lector literario, Antonio Mendoza (coord.), págs. 73-Ioo. Barcelona: Octaedro.

- Moreno Hernández, C. (1998). Literatura e Hipertexto. De la cultura manuscrita a la cultura electrónica. Madrid: UNED.

- Mcluhan, M. (2009). Comprender los medios de comunicación: las extensiones del ser humano. Barcelona: Paidós.

- Moulthrop, S. (2003). “El hipertexto y la política de la interpretación”. En María José Vega (Ed.), Literatura hipertextualy teoría literaria, págs. 23-3r. Madrid: Marenostrum.

- Orozco Vera, M. a J. (2005). "Literatura e hipertexto: hacia una lectura creativa". En Comunicación, universo artístico y nuevas tecnologías, María Elena Barroso Villar (ed.), 95-II2. Sevilla: Alfar.

- Pajares Tosca, S. (I998). http://www.ucm.es/info/especulo/hipertul/condex.html

-___-_(2004). Literatura Digital. El paradigma hipertextual. Cáceres: Universidad de Extremadura.

- Riesman, D. (1950). The Lonely Crowd. A Study of the Changing American Character. New Haven: Yale U.P.

- Romera Castillo, J. (ı996). “Literatura y nuevas tecnologías”. En José Romera Castillo et alii (Eds.), Literatura y multimedia, págs. I3-82. Madrid: Visor Libros.

- Romero López, D. y Sanz Cabrerizo, A. (Eds.) (2008). Literaturas del texto al hipermedia, Barcelona: Anthropos.

- Ruiz Domínguez, M. a M. (2OI2). "Hipertextos, metaficción y fantasía, en Corazón de Tinta”. En Leer hipertextos, Antonio Mendoza (Coord.), págs. I49-I34. Barcelona: Octaedro.

- Ryan, M.L. (2004). La narración como realidad virtual. La inmersión y la interactividad en la literature y en los medios electrónicos. Barcelona: Paidós.

- Sánchez-Mesa Martínez, D. (2OII). "Literatura aumentada. Intermedialidad/transmedialidad o el Viaje de Alicia a través de las pantallas”. En Literatura e Internet. Nuevos textos, nuevos lectores, Salvador Montesa (dir.), págs. Io9-I29. Málaga: AEDILE.

- Scolari, C. A. (2013). Narrativas transmedia. Cuando todos los medios cuentan. Barcelona: Deusto. 
- Stalin, J. M. (I990). "Reading hypertext: order an coherence in a new medium”. En Hypermedia and Literary Studies, Paul Delany y George P. Landow. Cambridge: The Mit Press, I99I.

- Tabernero, R. (20I2). "La hipertextualidad como fundamento de construcción en la literatura "iluminada": La formación del lector en el libro-álbum y en el libro ilustrado". En Leer hipertextos, Antonio Mendoza (Coord.), págs. I2I-I34. Barcelona: Octaedro.

- Talens, J. (2008). “Cultura, poesía y política en la era digital”. En Escrituras Digitales: Tecnologías de la creación en la era virtual, Virgilio Tortosa (ed.), 37-49. Alicante: Universidad de Alicante.

- Tortosa, V. (2008). “Una nueva lógica escritural: El hipertexto”. En Escrituras digitales. Tecnologías de la creación en la era virtual, Virgilio Tortosa (ed.), 5-97. Alicante: Universidad. 